\title{
Benzodiazepine modulation of $\mathrm{GABA}_{\mathrm{A}}$ receptor opening frequency depends on activation context: A patch clamp and simulation study
}

\author{
Matt T. Bianchi ${ }^{a, 1}$, Emmanuel J. Botzolakis ${ }^{b, 1}$, Andre H. Lagrange ${ }^{c}$, and Robert L. \\ Macdonald ${ }^{\mathrm{C}, \mathrm{d}, \mathrm{e},{ }^{*}}$ \\ a Sleep Disorders Center, Beth Israel Deaconess Medical Center, Boston, MA, United States \\ b Department of Neuroscience, Vanderbilt University School of Medicine, Nashville, TN, United \\ States \\ c Department of Neurology, Vanderbilt University School of Medicine, Nashville, TN, United States \\ d Department of Molecular Physiology and Biophysics, Vanderbilt University School of Medicine, \\ Nashville, TN, United States \\ e Department of Pharmacology, Vanderbilt University School of Medicine, Nashville, TN, United \\ States
}

\section{Summary}

Benzodiazepines (BDZs) are $\mathrm{GABA}_{\mathrm{A}}$ receptor modulators with anxiolytic, hypnotic, and anticonvulsant properties. BDZs are understood to potentiate $\mathrm{GABA}_{\mathrm{A}}$ receptor function by increasing channel opening frequency, in contrast to barbiturates, which increase channel open duration. However, the in vitro evidence demonstrating increased opening frequency involved prolonged exposure to sub-saturating GABA concentrations, conditions most similar to those found in extrasynaptic areas. In contrast, synaptic $\mathrm{GABA}_{\mathrm{A}}$ receptors are transiently activated by high GABA concentrations. To determine if BDZ modulation of single-channel opening frequency would be different for BDZ-sensitive receptors activated under synaptic versus extrasynaptic conditions, a combination of patch clamp recording and kinetic modeling was used. Consistent with the original experimental findings, BDZs were found to increase receptor affinity for GABA by decreasing the unbinding rate. While this mechanism was predicted to increase opening frequency under extrasynaptic conditions, simulations predicted that the same mechanism under synaptic conditions would increase the number, but not the frequency, of single-channel openings. Thus, a single mechanism (slower GABA unbinding) can produce differential changes in opening frequency under synaptic versus extrasynaptic conditions. The functional impact of BDZs on $\mathrm{GABA}_{\mathrm{A}}$ receptors therefore depends upon the physiological context of receptor activation.

\section{Keywords}

Mechanism; Ion channel; Synaptic; Extrasynaptic; Affinity

\footnotetext{
*Corresponding author at: Department of Neurology, Vanderbilt University Medical Center, 6140 Medical Research Building III, 465 21st Ave, South Nashville, TN 37232-8552, United States. Tel.: +1 615936 2287; fax: +1 6153225517. robert.macdonald@vanderbilt.edu, sandra.camp@ vanderbilt.edu (R.L. Macdonald).

${ }^{1}$ MTB and EJB contributed equally to the work.

Conflicts of interest

The authors report no conflicts of interest.
} 


\section{Introduction}

As the predominant source of fast synaptic inhibition in the brain, $\mathrm{GABA}_{\mathrm{A}}$ receptors have been the focus of emerging hypotheses not only of seizure pathophysiology, but also of anticonvulsant drug pharmacology. However, their role as the therapeutic target of benzodiazepines (BDZs) remained controversial for some time. While BDZs were observed to enhance $\mathrm{GABA}_{\mathrm{A}}$ receptor currents during intracellular recording from cultured neurons (Choh et al., 1977; Macdonald and Barker, 1978), others studies suggested that BDZs were antagonists of $\mathrm{GABA}_{\mathrm{A}}$ receptors (Gahwiler, 1976; Mathers, 1987), and still others indicated actions at glycine receptors (Young et al., 1974). Not until the development of single-channel recording (Colquhoun, 1991) was direct evidence presented that BDZs enhanced $\mathrm{GABA}_{\mathrm{A}}$ receptor responses (Rogers et al., 1994; Twyman et al., 1989). Specifically, BDZs were reported to increase single-channel opening frequency without altering mean open duration, an effect attributed to an increased affinity of GABA for the receptor (Rogers et al., 1994). Barbiturates, in contrast, were reported to increase the duration of openings without increasing their frequency (MacDonald et al., 1989; Twyman et al., 1989).

However, the in vitro electrophysiological evidence demonstrating BDZ-mediated increased opening frequency involved conditions most similar to those occurring in extrasynaptic areas, where receptors are persistently activated by sub-saturating concentrations of ambient GABA ( $\sim 1 \mu \mathrm{M}$ or less) (Farrant and Nusser, 2005). Under these conditions, binding of GABA to the receptor is infrequent, and because GABA is always present, receptors are free to repeatedly bind and unbind GABA. Synaptic receptors, in contrast, are transiently activated ( 1 ms) by saturating concentrations of GABA $(\sim 1 \mathrm{mM})$ (Jones and Westbrook, 1996), such that receptors are predominantly GABA-bound, and the majority of channel activity (which can last 10-100 $\mathrm{s}$ of milliseconds) occurs without the prospect of rebinding. Given that BDZs were reported to increase the affinity of GABA for the receptor, a mechanism that favors GABA-bound receptor conformations, this suggests that the effects of BDZs on opening frequency would be different for receptors activated under synaptic versus extrasynaptic conditions. Although extrasynaptic receptors are enriched for BDZ-insensitive receptor isoforms (i.e., those containing the $\delta$ subunit), BDZ-sensitive receptor isoforms (i.e., those containing the $\gamma$ subunit) occur commonly in extrasynaptic locations as well (Glykys et al., 2008; Glykys and Mody, 2007), and in some cases, are actually more abundant than BDZ-insensitive receptors (Farrant and Nusser, 2005). Therefore, the potential context-dependence of BDZ modulation of $\mathrm{GABA}_{\mathrm{A}}$ receptor function remains an important mechanistic issue.

\section{Materials and methods}

\section{Cell culture and electrophysiology}

HEK293T cells transiently expressing $\alpha 1, \beta 3$, and $\gamma 2 \mathrm{~L} \mathrm{GABA}_{\mathrm{A}}$ receptor subunit cDNAs were prepared for patch clamp electrophysiological recordings as described previously (Bianchi and Macdonald, 2001). Briefly, whole cell patch clamp experiments involved gently lift the patched cells from the culture dish. Solutions containing GABA (with or without diazepam) were then applied to these lifted cells using a rapid drug application system composed of 4-barrelled square glass capillary tubing (Hinkle et al., 2003). The solution interface was translated across the cell using a mechanical stepper device (Warner Instruments, USA). Solution exchange times were consistently $<400 \mu \mathrm{s}$ when measured at the open patch electrode, but were slightly slower around the lifted cell.

\section{Kinetic simulations}

Simulations of macroscopic currents (100 s of receptors) were conducted using Berkeley Madonna (www.berkeleymadonna.com) software that solves the probability that a receptor 
will occupy any state of a kinetic model as a function of time. QUB (www.qub.buffalo.edu) software was used for single-channel simulations such that resting $\left(C_{\mathrm{u}}\right)$, bound closed $\left(C_{\mathrm{b}}\right.$ and $D$ ), and bound open $(O)$ states could be distinguished via assigning different (arbitrary) current levels ( $C_{\mathrm{u}}=0$ units, $C_{\mathrm{b}}$ and $D=-2$ units, and $O=-6$ units, respectively) to each state. For equilibrium ("extrasynaptic") simulations, $10 \mathrm{~ms}$ after the start of the trial, $1 \mu \mathrm{M}$ GABA was applied for $1990 \mathrm{~ms}$. 10 such trials constituted a batch. For phasic ("synaptic") simulations, 10 $\mathrm{ms}$ after the start of a trial, $1 \mathrm{mM}$ GABA was applied for $1 \mathrm{~ms}$. Channel openings were then observed for $490 \mathrm{~ms}$. 50 such trials were analyzed per batch, and batches were averaged for analysis. GraphPad Prism (www.graphpad.com) was used for statistical analysis. All data were plotted as mean \pm SEM, and statistical significance was taken as $p<0.05$ using ANOVA followed by Tukey post hoc analysis.

\section{Results}

\section{$\mathrm{GABA}_{A}$ receptor macroscopic current properties were predicted to have different sensitivities to changes in GABA affinity}

BDZs have been shown to enhance $\mathrm{GABA}_{\mathrm{A}}$ receptor currents by increasing the affinity of GABA for the receptor (which can involve decreasing the unbinding or increasing the binding rate constants) (Twyman et al., 1989), causing a "left shift" of the GABA concentrationresponse curve (Ghansah and Weiss, 1999). This mechanism predicts that BDZs should increase peak current amplitude only when receptors are activated by sub-saturating concentrations of GABA. Once receptors are activated by a saturating GABA concentration, no further increase in peak current should be possible through increasing agonist binding affinity (Ghansah and Weiss, 1999). Furthermore, desensitization (the loss of current in the continued presence of agonist) should not be affected by BDZs during application of a saturating concentration of GABA, again because binding sites are already saturated (Bianchi et al., 2007). In contrast, the sensitivity of deactivation (the process by which currents return to baseline following GABA washout) to BDZs should depend on whether BDZs increased affinity by increasing the binding rate or decreasing the unbinding rate. Since deactivation occurs in the absence of free GABA, its time-course should be insensitive to changes in the binding rate (defined as the product of the "true" binding rate and the GABA concentration, which equals zero during deactivation). In contrast, deactivation should be highly sensitive to slower unbinding, which increases the average time receptors spend GABA-bound, thus allowing for additional openings.

These predictions were tested using a simple kinetic model of $\mathrm{GABA}_{\mathrm{A}}$ receptor function, previously shown to be useful for investigating the relationship between microscopic and macroscopic current kinetics (Fig. 1) (Bianchi et al., 2007) While this simplified model does not capture the rich kinetic behavior of $\mathrm{GABA}_{\mathrm{A}}$ receptors (Haas and Macdonald, 1999), it provides qualitative representation of the salient features of $\mathrm{GABA}_{\mathrm{A}}$ receptor currents (fast activation, fast desensitization, and relatively slow deactivation). In this model, receptors can occupy one of 4 states: a resting closed state (closed-unbound, $C_{\mathrm{u}}$ ), a GABA-bound closed state (closed-bound, $C_{\mathrm{b}}$ ), a GABA-bound open state (open, $O$ ), and an additional more stable GABA-bound closed state that permits desensitization to occur (desensitized, $D$ ). As predicted, increasing affinity by increasing $K_{\text {on }}$ (Fig. 2A) or decreasing $K_{\text {off }}$ (Fig. 2B) increased peak amplitude when currents were evoked by a sub-saturating GABA concentration (Fig. 2A1 and B1), but neither did so when currents were evoked by a saturating GABA concentration (Fig. $2 \mathrm{~A} 2$ and $\mathrm{B} 2$ ). Desensitization occurring in the presence of a saturating GABA concentration was also not affected by increased affinity, either by increasing $K_{\text {on }}$ or decreasing $K_{\text {off }}$ (Fig. $2 \mathrm{~A} 2$ and B2). Deactivation, in contrast, was highly sensitive to increases in affinity, even in the context of saturating GABA, but only by decreasing $K_{\text {off }}$. This was true for deactivation following prolonged exposures to GABA (Fig. 2A2 and B2) and also for deactivation following 
"synaptic" (1 ms) pulses (Fig. 2A3 and B3). Similar results were obtained using comprehensive models containing multiple open, closed, and desensitized states (Haas and Macdonald, 1999; Lagrange et al., 2007).

\section{BDZs enhanced GABA $A_{A}$ receptor currents by decreasing the GABA unbinding rate}

Although single-channel studies suggested that $\mathrm{BDZs}$ enhanced $\mathrm{GABA}_{\mathrm{A}}$ receptor currents by increasing GABA affinity, it remains unclear whether this is mediated by an increased rate of GABA binding or a decreased rate of GABA unbinding (Lavoie and Twyman, 1996). Therefore, guided by the kinetic simulations in the previous section, we reevaluated BDZmediated increase in GABA affinity using electrophysiolgy. Whole-cell patch clamp recordings were obtained from HEK293T cells transiently expressing $\alpha 1 \beta 3 \gamma 2 \mathrm{~L} \mathrm{GABA}_{\mathrm{A}}$ receptors (Fig. 3). GABA was applied with or without $1 \mu \mathrm{M}$ diazepam (DZP) using a rapid solution exchange system. DZP was pre-applied for $1 \mathrm{~s}$ to allow for equilibration (the slight baseline shift with BDZ pre-application may reflect a low-affinity direct activation mechanism) (Bianchi and Macdonald, 2001). Consistent with BDZs increasing the affinity of GABA for the receptor, DZP co-application increased the peak amplitude and desensitization of currents evoked by a sub-saturating concentration of GABA $\left(1 \mu \mathrm{M}, \sim \mathrm{EC}_{25}\right)$ (Fig. 3A), but had no effect on the amplitude or desensitization of currents evoked by a saturating concentration of GABA (3 mM, $\sim \mathrm{EC}_{99}$ ) (Fig. 3B). Deactivation, however, was always prolonged in the presence of DZP, for both low and high GABA concentrations (Fig. 3A and B), and for brief (5 ms) synaptic-like pulses of GABA (Fig. 3C). Taken together, these findings suggested that BDZs increased GABA affinity by decreasing the GABA unbinding rate, $K_{\text {off. }}$. Although we cannot exclude a mixed mechanism that involves both $K_{\text {on }}$ and $K_{\text {off }}$, the data are incompatible with BDZs modulating $K_{\text {on }}$ alone.

\section{Decreasing the GABA unbinding rate is predicted to increase opening frequency of $\mathrm{GABA}_{A}$ receptors activated under tonic "extrasynaptic" conditions}

Single-channel opening frequency is defined as the number of openings per unit time. Since individual openings must be flanked by closures, opening frequency is determined by the average time channels spend open and by the average time channels spend closed (this determines the average interval between openings). However, when channels are capable of accessing multiple closed states, as in the kinetic model shown in Fig. 1, the overall average closed time depends not only on the average lifetime of each individual closed state (which equals the reciprocal sum of the exit rates from each state), but also on the weighted likelihood of reaching each closed state (which is determined by a more complex relationship between rates) (Colquhoun and Hawkes, 1982). For receptors activated under extrasynaptic conditions (i.e., prolonged exposure to a sub-saturating concentration of GABA), the average lifetime of the $C_{\mathrm{u}}$ state is relatively long (since the effective binding rate is determined by the product of $K_{\text {on }}$ and the GABA concentration), and therefore, contributes substantially to the average closed time. By decreasing $K_{\text {off }}$, BDZs decrease the likelihood that receptors enter this longlived state, thus decreasing the average closed time. In other words, with slower unbinding, the average length of channel closure becomes dominated by the time spent in two closed states $\left(C_{\mathrm{b}}\right.$ and $\left.D\right)$ as opposed to three $\left(C_{\mathrm{u}}, C_{\mathrm{b}}\right.$, and $\left.D\right)$. Thus, BDZs are predicted to decrease the interval between openings and, therefore, increase opening frequency.

To evaluate this phenomenon explicitly, single-channel activity was simulated using the simple kinetic model (Fig. 1) under extrasynaptic conditions: a low concentration of GABA (1 $\mu \mathrm{M})$ applied for an extended period of time (2000 ms) (Fig. 4). Increasing BDZ concentration was simulated by varying $K_{\text {off }}$ from $1000 \mathrm{~s}^{-1}$ (baseline) to progressively lower values (600, 200, and $50 \mathrm{~s}^{-1}$ ). The "EC ${ }_{X}$ " notation denotes the GABA concentration producing $X$ percent of maximal current amplitude. Based on simulated concentration-response curves (not shown), $1 \mu \mathrm{M}$ GABA was approximately $\mathrm{EC}_{2}$ when $K_{\text {off }}$ was $1000, \mathrm{EC}_{15}$ when $K_{\text {off }}$ was $600, \mathrm{EC}_{25}$ 
when $K_{\text {off }}$ was 200 , and $\mathrm{EC}_{35}$ when $K_{\text {off }}$ was $50 \mathrm{~s}^{-1}$. The use of a simplified model and a single concentration of $\mathrm{BDZ}$ precluded direct comparison of the magnitude of BDZ modulation with that seen in the patch clamp experiments, although this would not alter the interpretations of our simulation data.

Unlike "real" single-channel recordings, where one cannot explicitly distinguish different states with the same conductance (such as different closed states), the simulations allowed us to unambiguously determine the receptor occupation of each state by assigning them different current levels (Fig. 4A; see Section 2). We were specifically interested in distinguishing the unbound state $\left(C_{\mathrm{u}}\right.$, level 1$)$ from the GABA-bound non-conducting states $\left(C_{\mathrm{b}}\right.$ and $D$, level 2$)$, and also from the single GABA-bound conducting state $(O$, level 3$)$. As expected, the transition profile in the presence of BDZ (lower trace) consisted of less time in the unbound closed state (level 1) and more time in the bound closed (level 2) and open (level 3) states than did the transition profile in the absence of BDZ (top trace). Note that in both cases channels spent only a fraction of the total GABA-bound time in the open state; the remainder of bound time was spent in $C_{\mathrm{b}}$ and $D$. Since decreasing the unbinding rate did not affect channel mean open time (not shown), this shift of receptor occupancy towards the GABA-bound conformations increased overall channel opening frequency (Fig. 4B).

However, when analysis was restricted to the time when receptors were GABA-bound (something that can only be accomplished in silico), opening frequency was not found to increase (Fig. 4C). This critical difference reflects the fact that the increase in overall frequency (which is what was measured in the previously reported experiments using prolonged exposure to low GABA concentrations) was mediated entirely by decreased occupancy of the unbound closed state $\left(C_{\mathrm{u}}\right)$. Therefore, when we eliminated this state from our calculations, the effect on opening frequency disappeared. In other words, the observed frequency change simply reflected the ability of receptors to bind and rebind GABA in the experimental conditions, as opposed to a fundamental change in receptor kinetics.

Interestingly, opening frequency actually tended to decrease with lower values of $K_{\text {off }}$. Although seemingly counterintuitive, this reflected the fact that decreasing the unbinding rate actually increased the average lifetime of the $C_{\mathrm{b}}$ state, which partly determined the GABAbound closed time. The magnitude of this decrease in frequency was small simply because the average lifetime of the $C_{\mathrm{b}}$ state was short compared to that of the $D$ state, which dominated the GABA-bound closed time.

\section{Decreasing the unbinding rate is predicted to increase the number, but not the frequency, of single-channel openings under phasic "synaptic" conditions}

Synaptic receptors are activated under markedly different conditions from those traditionally used to evaluate the effects of BDZs on single-channels, suggesting that BDZs will have a different effect on their opening frequency. Indeed, if receptors are fully saturated by vesicular release of GABA, then receptor occupancy in $C_{\mathrm{u}}$ approaches zero. This limits the ability of BDZs to affect opening frequency, as decreasing the unbinding rate cannot further increase the fraction of receptors that are GABA-bound (a "ceiling" effect). Importantly, this inability to affect opening frequency is independent of the duration of the GABA transient: as long as the GABA concentration is high, the $C_{\mathrm{u}}$ species is negligible, and thus frequency will not be increased whether the GABA transient is $1 \mathrm{~ms}$ (as in our simulations) or $5 \mathrm{~ms}$ (as in our experiments). Although our simulations and experiments do not address the potential contributions of altered diffusion and/or transporter function on the time-course of the GABA transient, it should be noted that even if receptors are not saturated, once GABA is cleared from the synaptic cleft, all subsequent openings shaping the synaptic current necessarily reflect transitions among GABA-bound states (i.e., $C_{\mathrm{b}}, D$, and $O$ ). Our results, however, demonstrated that decreasing the unbinding rate cannot increase opening frequency when channel activity 
does not involve the $C_{\mathrm{u}}$ state (Fig. 4), suggesting that BDZs would not increase opening frequency under synaptic conditions.

To test this hypothesis, single-channel responses to $1 \mathrm{~ms}$ pulses of $1 \mathrm{mM} \mathrm{GABA}$ were simulated (Fig. 5). As in the previous section, $K_{\text {off }}$ was set to $1000 \mathrm{~s}^{-1}$ in the baseline condition, and increasing BDZ concentrations were simulated using progressively lower $K_{\text {off }}$ values (600, 200, and $50 \mathrm{~s}^{-1}$ ) (Fig. 5A). Unbinding (entry into $C_{\mathrm{u}}$ ) was considered a terminal event for calculations of opening frequency, since we assumed that rebinding was negligible under synaptic conditions. Opening frequency was therefore defined as the number of openings divided by the time spent GABA-bound. Note that being able to determine precisely the moment of unbinding overcomes a critical limitation for testing this hypothesis experimentally, where unbinding is not associated with a change in channel conductance and thus cannot be measured or inferred. In other words, with "real" experiments, one cannot justify how long to "watch" for openings after a GABA pulse is delivered, yet this duration specifies the denominator for calculations of opening frequency. As expected, neither the open durations (Fig. 5B) nor the opening frequency (Fig. 5C) were increased by decreasing the unbinding rate. However, slower unbinding increased the average time receptors spent GABA-bound (Fig. 5D), which increased the total number of openings (Fig. 5E). Thus, under synaptic conditions, BDZs are predicted to increase the number of channel openings without increasing opening frequency.

\section{Discussion}

The unique conditions of synaptic transmission prevent BDZs from increasing singlechannel opening frequency

Electrophysiological studies have demonstrated that BDZs increase single-channel opening frequency by increasing GABA affinity (Rogers et al., 1994; Twyman et al., 1989). However, these experiments were performed using low concentrations of GABA, which allowed channels to spend considerable time in the unbound closed state $\left(C_{\mathrm{u}}\right)$, and prolonged exposure to GABA, which allowed channels to repeatedly access this state (i.e., bind and unbind GABA). In this context, which resembles extrasynaptic transmission in vivo, BDZs were able to increase opening frequency by decreasing the contribution of the $C_{\mathrm{u}}$ state to the overall average closed time. This state, however, plays a minimal role in opening frequency when receptors are activated under synaptic conditions for two main reasons. First, receptor occupancy in $C_{\mathrm{u}}$ rapidly approaches zero with the high concentrations of GABA reached in synapses. Second, the high rate at which free GABA is cleared from the synaptic cleft renders rebinding (and therefore, the contribution of the $C_{\mathrm{u}}$ state to the average closed interval) minimal under synaptic conditions, even if synaptic $\mathrm{GABA}_{\mathrm{A}}$ receptors are not saturated (Perrais and Ropert, 1999; Poncer et al., 1996). Thus, BDZs are not predicted to increase the single-channel opening frequency of synaptic $\mathrm{GABA}_{\mathrm{A}}$ receptors.

\section{BDZs enhance synaptic currents by increasing the number of single-channel openings}

If BDZs cannot increase single-channel opening frequency of receptors activated under synaptic conditions, what then explains their ability to enhance synaptic currents (Nusser and Mody, 2002)? Our simulations demonstrated that by delaying GABA unbinding, BDZs increase the average time receptors are GABA-bound, thus increasing the total number of channel openings following a brief pulse of GABA. Indeed, once a receptor enters the $C_{\mathrm{b}}$ state, the likelihood of unbinding GABA versus continuing to transition among GABA-bound states is determined by the ratio of the unbinding rate $\left(K_{\mathrm{off}}\right)$ to the sum of the alternatives (entry into $O$ and $D$ ). Thus, by decreasing $K_{\text {off }}$, BDZs extend the window of time during which individual receptors can transition among GABA-bound states, prolonging synaptic currents. Unlike stabilizing desensitized states, which also prolongs synaptic currents by increasing the average 
time receptors are GABA-bound (Bianchi et al., 2007; Jones and Westbrook, 1995), delaying unbinding by decreasing $K_{\text {off }}$ necessarily increases synaptic charge transfer. This is because decreasing $K_{\text {off }}$ increases the number of channels available for opening at each moment in time after the GABA pulse (i.e., the "population" opening frequency increases), which increases the total number of openings.

\section{BDZs are predicted to increase the single-channel opening frequency of extrasynaptic $\mathrm{GABA}_{A}$ receptors}

Increasing evidence suggests that extrasynaptic $\mathrm{GABA}_{\mathrm{A}}$ receptors make important contributions to inhibitory signaling (Kullmann et al., 2005). In contrast to synaptic signaling that is brief, point-to-point, and involves high neurotransmitter concentrations, extrasynaptic communication involves low and slowly changing concentrations of neurotransmitters diffusing over larger distances than the synaptic cleft (Kullmann et al., 2005). Although the physiological relevance of extrasynaptic transmission remains poorly understood, the effects of BDZs observed on single-channel currents evoked under equilibrium conditions may prove relevant for understanding how this subset of $\mathrm{GABA}_{\mathrm{A}}$ receptors are modulated by BDZs. While the majority of extrasynaptic receptors include the $\delta$ subunit, which renders them insensitive to BDZs (McKernan and Whiting, 1996; Mehta and Ticku, 1999), $\gamma$ subunit-containing receptors sensitive to the effects of BDZs have also been found outside synapses in a number of brain regions including the hippocampus (Glykys and Mody, 2007). Our simulations predicted that BDZs would in fact increase single-channel opening frequency of BDZ-sensitive extrasynaptic receptors. Although the relative importance of synaptic and extrasynaptic $\mathrm{GABA}_{\mathrm{A}}$ receptors to neurological diseases such as epilepsy remains uncertain, the dependence of BDZ modulation on activation context may have important relevance for anticonvulsant treatment. For example, BDZs may prolong synaptic currents in situations in which they are pathologically brief such as the $\gamma 2$ subunit mutation, K289M, associated with generalized epilepsy with febrile seizures plus (Bianchi et al., 2002). It is conceivable that extrasynaptic receptors (which may depend upon ambient rather than synaptic GABA) can be modulated by BDZs independent of synaptic inhibition or when synaptic inhibition is pathologically compromised.

\section{Single-channel opening frequency is sensitive to multiple kinetic mechanisms}

Although single-channel opening frequency is a relatively straightforward experimental measurement, there are in fact a variety of "mechanisms" that influence opening frequency. For example, just as decreasing $K_{\text {off }}$ can increase opening frequency by decreasing the average channel closed time (under extrasynaptic conditions), frequency can also be increased by decreasing the entry rate or increasing the exit rate from the desensitized state. Interestingly, opening frequency can also be increased by decreasing the time spent in the open state (by increasing the exit rate $O^{-}$), as this will decrease the length of each open-closed cycle. However, increasing open frequency in this manner will actually decrease overall channel open probability. Thus, changes in opening frequency alone can neither specify a kinetic mechanism nor provide information regarding channel effectiveness (the open duration must also be known). This distinction between observation (opening frequency) and mechanism (increased affinity via $K_{\text {off }}$ ) forms the basis for our demonstration of "context-dependent" BDZ modulation of $\mathrm{GABA}_{\mathrm{A}}$ receptor opening frequency. Thus, a single mechanism (increased affinity via decreasing $K_{\text {off }}$ ) yields different functional impacts on opening frequency (and macroscopic current properties) depending on the context in which receptors are activated. Despite this kinetic difference, $\mathrm{GABA}_{\mathrm{A}}$ receptor function is enhanced by BDZs in both synaptic and extrasynaptic contexts. Although detailed kinetic models of most $\mathrm{GABA}_{\mathrm{A}}$ receptor isoforms have not been established, the mechanistic arguments of this study are likely to apply in general to BDZ-sensitive isoforms, because they hold for any model in which 
binding precedes transitions to open, pre-open and desensitized states, regardless of their number or connectivity.

\section{Acknowledgments}

Funded by NIH grant NS33300 to RLM

\section{References}

Bianchi MT, Botzolakis EJ, Haas KF, Fisher JL, Macdonald RL. Microscopic kinetic determinants of macroscopic currents: insights from coupling and uncoupling of $\mathrm{GABA}_{\mathrm{A}}$ receptor desensitization and deactivation. J Physiol 2007;584:769-787. [PubMed: 17884921]

Bianchi MT, Macdonald RL. Agonist trapping by GABAA receptor channels. J Neurosci 2001;21:90839091. [PubMed: 11717341]

Bianchi MT, Song L, Zhang H, Macdonald RL. Two different mechanisms of disinhibition produced by $\mathrm{GABA}_{\mathrm{A}}$ receptor mutations linked to epilepsy in humans. J Neurosci 2002;22:5321-5327. [PubMed: 12097483]

Choh DW, Farb DH, Fischbach GD. Chlordiazepoxide selectively augments GABA action in spinal cord cell cultures. Nature 1977;269:342-344. [PubMed: 561893]

Colquhoun D. Neher and Sakmann win Nobel Prize for patch-clamp work. Trends Pharmacol Sci 1991;12:449. [PubMed: 1724328]

Colquhoun D, Hawkes AG. On the stochastic properties of bursts of single ion channel openings and of clusters of bursts. Philos Trans R Soc London B: Biol Sci 1982;300:1-59. [PubMed: 6131450]

Farrant M, Nusser Z. Variations on an inhibitory theme: phasic and tonic activation of GABA(A) receptors. Nat Rev Neurosci 2005;6:215-229. [PubMed: 15738957]

Gahwiler BH. Diazepam and chlordiazepoxide: powerful GABA antagonsits in explants of rat cerebellum. Brain Res 1976;107:176-179. [PubMed: 1268721]

Ghansah E, Weiss DS. Benzodiazepines do not modulate desensitization of recombinant alpha1beta2gamma2 GABA(A) receptors. Neuroreport 1999;10:817-821. [PubMed: 10208554]

Glykys J, Mann EO, Mody I. Which GABA(A) receptor subunits are necessary for tonic inhibition in the hippocampus? J Neurosci 2008;28:1421-1426. [PubMed: 18256262]

Glykys J, Mody I. Activation of GABA $\mathrm{A}_{\mathrm{A}}$ receptors: views from outside the synaptic cleft. Neuron 2007;56:763-770. [PubMed: 18054854]

Haas KF, Macdonald RL. GABA $A_{A}$ receptor subunit gamma2 and delta subtypes confer unique kinetic properties on recombinant $\mathrm{GABA}_{\mathrm{A}}$ receptor currents in mouse fibroblasts. J Physiol 1999;514 (Pt 1):27-45. [PubMed: 9831714]

Hinkle DJ, Bianchi MT, Macdonald RL. Modifications of a commercial perfusion system for use in ultrafast solution exchange during patch clamp recording. Biotechniques 2003;35:472-474. 476. [PubMed: 14513550]

Jones MV, Westbrook GL. Desensitized states prolong GABA $\mathrm{A}$ channel responses to brief agonist pulses. Neuron 1995;15:181-191. [PubMed: 7542462]

Jones MV, Westbrook GL. The impact of receptor desensitization on fast synaptic transmission. Trends Neurosci 1996;19:96-101. [PubMed: 9054063]

Kullmann DM, Ruiz A, Rusakov DM, Scott R, Semyanov A, Walker MC. Presynaptic, extrasynaptic and axonal $\mathrm{GABA}_{\mathrm{A}}$ receptors in the CNS: where and why? Prog Biophys Mol Biol 2005;87:33-46. [PubMed: 15471589]

Lagrange AH, Botzolakis EJ, Macdonald RL. Enhanced macroscopic desensitization shapes the response of alpha4 subtype-containing $\mathrm{GABA}_{\mathrm{A}}$ receptors to synaptic and extrasynaptic $\mathrm{GAB}_{\mathrm{A}}$. J Physiol 2007;578:655-676. [PubMed: 17124266]

Lavoie AM, Twyman RE. Direct evidence for diazepam modulation of $\mathrm{GABA}_{\mathrm{A}}$ receptor microscopic affinity. Neuropharmacology 1996;35:1383-1392. [PubMed: 9014155]

Macdonald R, Barker JL. Benzodiazepines specifically modulate GABA-mediated postsynaptic inhibition in cultured mammalian neurones. Nature 1978;271:563-564. [PubMed: 563985]

Epilepsy Res. Author manuscript; available in PMC 2010 August 1. 
MacDonald RL, Rogers CJ, Twyman RE. Barbiturate regulation of kinetic properties of the GABAA receptor channel of mouse spinal neurones in culture. J Physiol 1989;417:483-500. [PubMed: 2482885]

Mathers DA. The GABA receptor: new insights from single-channel recording. Synapse 1987;1:96101. [PubMed: 2905528]

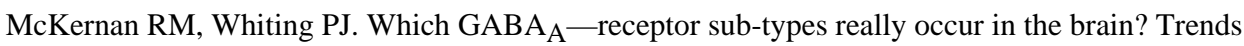
Neurosci 1996;19:139-143. [PubMed: 8658597]

Mehta AK, Ticku MK. An update on GABA_ receptors. Brain Res Brain Res Rev 1999;29:196-217. [PubMed: 10209232]

Nusser Z, Mody I. Selective modulation of tonic and phasic inhibitions in dentate gyrus granule cells. J Neurophysiol 2002;87:2624-2628. [PubMed: 11976398]

Perrais D, Ropert N. Effect of zolpidem on miniature IPSCs and occupancy of postsynaptic GABAA receptors in central synapses. J Neurosci 1999;19:578-588. [PubMed: 9880578]

Poncer JC, Durr R, Gahwiler BH, Thompson SM. Modulation of synaptic GABA A receptor function by benzodiazepines in area CA3 of rat hippocampal slice cultures. Neuropharmacology 1996;35:11691179. [PubMed: 9014132]

Rogers CJ, Twyman RE, Macdonald RL. Benzodiazepine and beta-carboline regulation of single GABA $_{A}$ receptor channels of mouse spinal neurones in culture. J Physiol 1994;475:69-82. [PubMed: 7514665]

Twyman RE, Rogers CJ, Macdonald RL. Differential regulation of gamma-aminobutyric acid receptor channels by diazepam and phenobarbital. Ann Neurol 1989;25:213-220. [PubMed: 2471436]

Young AB, Zukin SR, Snyder SH. Interaction of benzodiazepines with central nervous glycine receptors: possible mechanism of action. Proc Natl Acad Sci USA 1974;71:2246-2250. [PubMed: 4152296]

Epilepsy Res. Author manuscript; available in PMC 2010 August 1. 

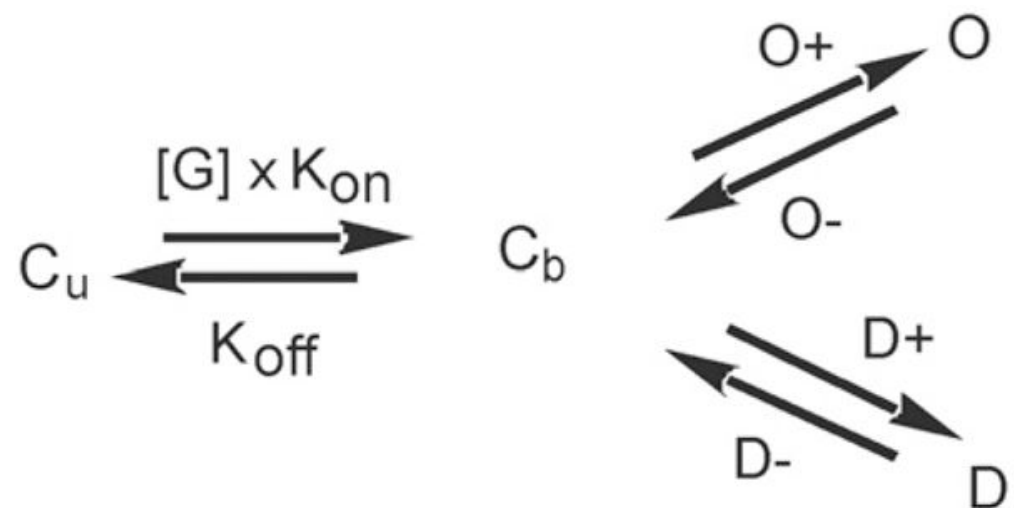

Figure 1.

Simplified 4-state kinetic model of $\mathrm{GABA}_{\mathrm{A}}$ receptor ion channel function. The model contains a single unbound closed state $\left(C_{\mathrm{u}}\right)$. Agonist binding initiates the transition from $C_{\mathrm{u}}$ to the bound closed state $\left(C_{\mathrm{b}}\right)$. This state can reversibly transition into either the open $(O)$ or the desensitized $(D)$ state. Note that the binding rate is the product of $K_{\text {on }}$ and the GABA concentration, which have units of $\mathrm{M}^{-1} \mathrm{~s}^{-1}$ and $\mathrm{M}$, respectively. The remaining rate constants have units of $\mathrm{s}^{-1}$. Model rate constants are: $K_{\mathrm{on}}=5 \times 106 ; K_{\mathrm{off}}=1000 ; O+=800 ; O-=500 ; D+=800 ; D^{-}=$ 100. 

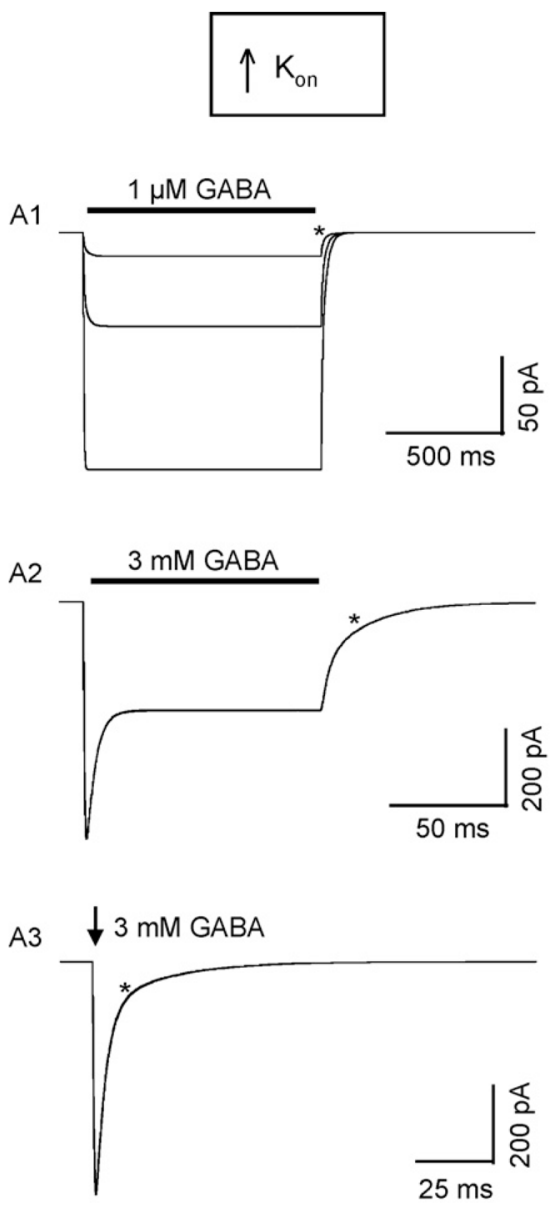
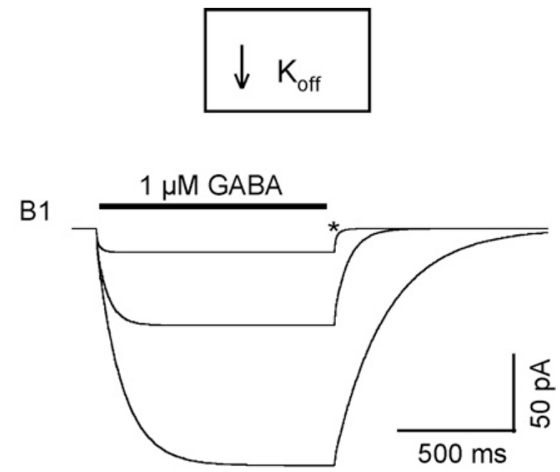

B2

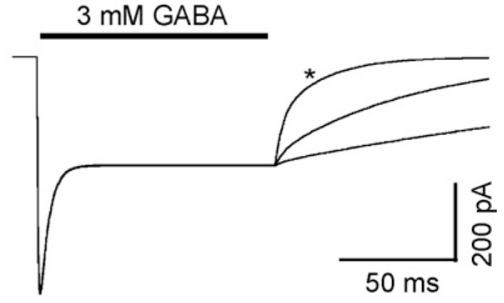

B3

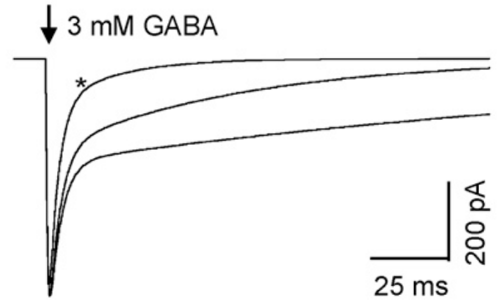

Figure 2.

The effect of increasing GABA affinity by increasing $K_{\text {on }}$ or decreasing $K_{\text {off }}$ on macroscopic current properties. Increasing receptor affinity for GABA can be accomplished either by increasing $K_{\text {on }}$ (left column) or decreasing $K_{\text {off (right column). (A) Increasing GABA affinity }}$ by increasing $K_{\mathrm{on}}$ increased current amplitudes in the context of a sub-saturating $(1 \mu \mathrm{M}$; $\sim \mathrm{EC}_{2}$ ) GABA concentration (panel (A1)), but had no appreciable effect on currents evoked by a saturating (3 mM; $\sim \mathrm{EC}_{99}$ ) GABA concentration, either after long (panel (A2)) or short (panel (A3)) duration GABA applications. The asterisk $(*)$ indicates the baseline current $\left(K_{\mathrm{on}}=5 \times\right.$ $106)$, and currents evoked in the context of progressively higher values of $K_{\text {on }}(2.5 \times 107$ and $1 \times 108$ ) were superimposed. (B) Increasing GABA affinity by decreasing $K_{\text {off }}$ also increased current amplitudes in the context of a sub-saturating GABA concentration (panel (B1)) but not in the context of a saturating GABA concentration (panels (B2 and B3)). However, deactivation was substantially prolonged with decreasing $K_{\text {off }}$ at all GABA concentrations, independent of the application duration. The asterisk $(*)$ indicates the baseline current $\left(K_{\mathrm{off}}=1000\right)$, and currents evoked in the context of progressively lower values of $K_{\text {on }}$ (200 and 50) were superimposed. Currents are downward going by convention. 


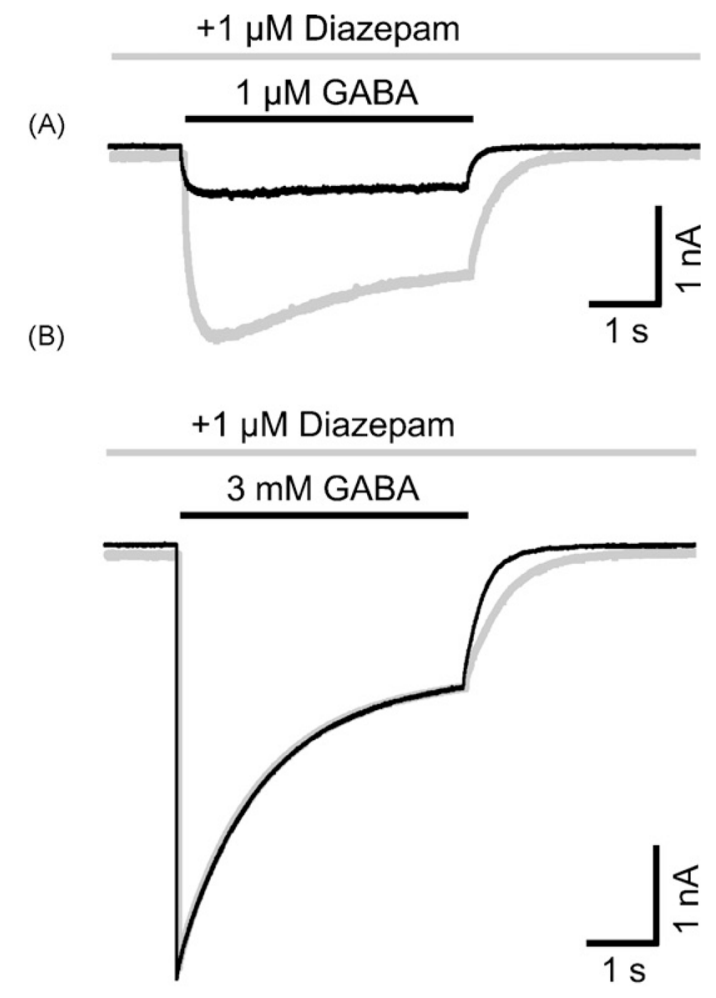

(C)

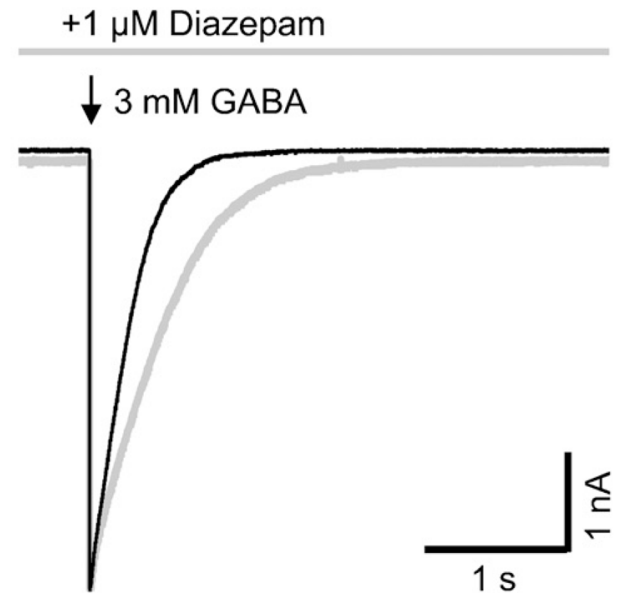

Figure 3.

Modulation of $\mathrm{GABA}_{\mathrm{A}}$ receptor currents by diazepam. (A) Currents evoked from cells expressing $\alpha 1 \beta 3 \gamma 2 \mathrm{~L} \mathrm{GABA}_{\mathrm{A}}$ receptors by a sub-saturating GABA concentration $(1 \mu \mathrm{M}$; black line) were enhanced by concurrent application of diazepam ( $1 \mu \mathrm{M}$; gray line). (B) Similar experimental protocol as in panel (A), using a saturating GABA concentration (3 mM). Note that the current amplitude and shape of desensitization (i.e., during the GABA application; black bar above traces) were unchanged by diazepam. In contrast, current deactivation (i.e., after the GABA application) was prolonged by diazepam. (C) Diazepam prolonged deactivation after a brief ( $5 \mathrm{~ms}$, "synaptic") pulse of saturating GABA. 
(A) Koff $=600 \mathrm{~s}^{-1}$
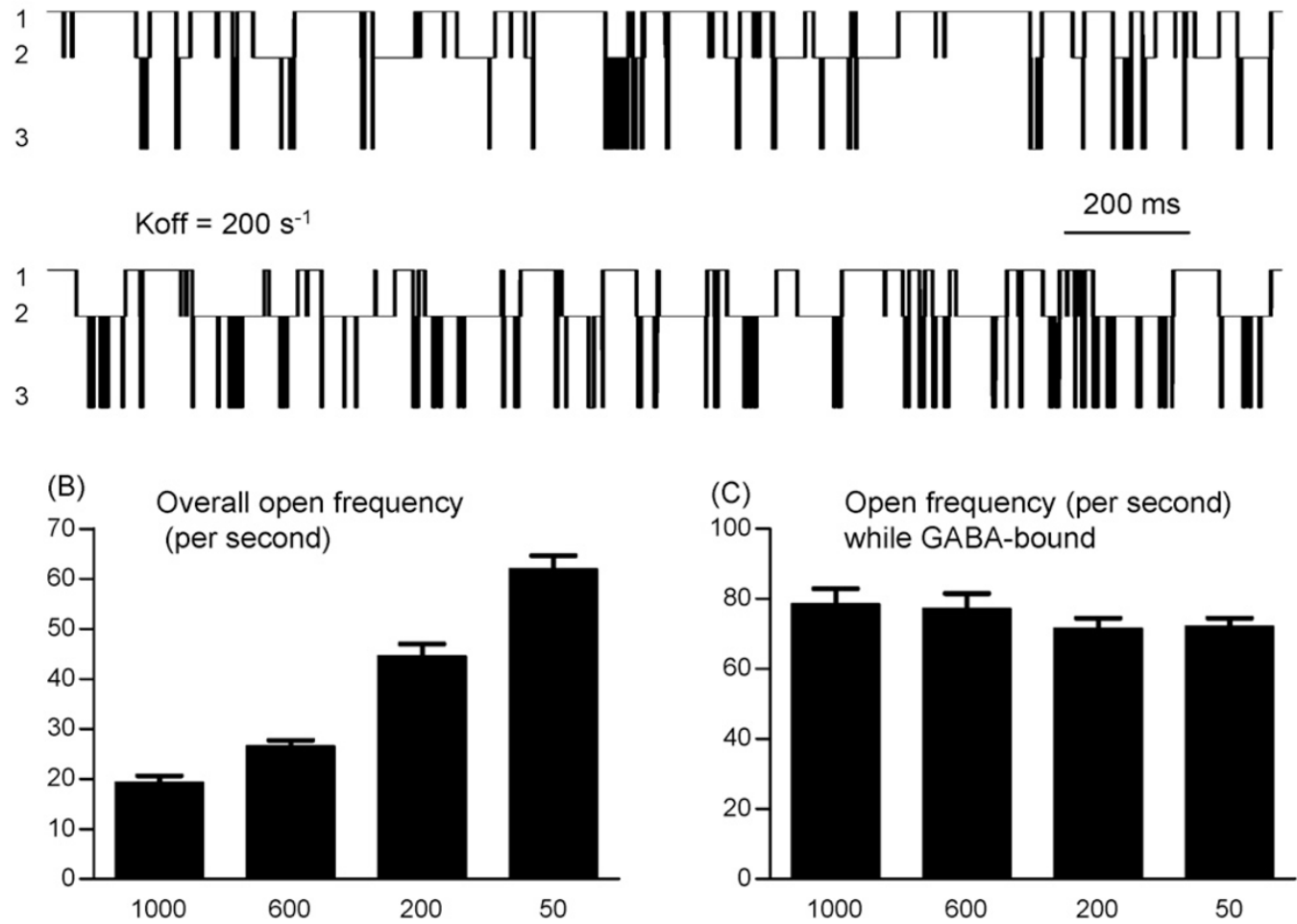

Figure 4.

Simulated single-channel activity under "extrasynaptic" conditions. (A) Simulated singlechannel activity in response to continuous exposure to $1 \mu \mathrm{M} \mathrm{GABA}$ when $K_{\mathrm{off}}$ is $600 \mathrm{~s}^{-1}$ (top trace) and when $K_{\text {off }}$ is $200 \mathrm{~s}^{-1}$ (bottom trace). The current levels designate receptor presence in the $C_{\mathrm{u}}$ (level 1), $C_{\mathrm{b}}$ or $D$ (level 2), and $O$ (level 3) states. Decreasing $K_{\text {off }}$ (which increases GABA affinity) leads to an increase in the frequency of openings (currents reaching the 3rd current level), and less time spent in the resting unbound state (the baseline current level). The horizontal time bar applies to both traces. (B) Overall opening frequency, including time spent in the unbound state, increased as $K_{\text {off }}$ decreased, with significant differences in comparison of every pair of columns. (C) Opening frequency, restricted to the time during which the channel was GABA-bound, did not increase as $K_{\text {off }}$ was decreased. In this and subsequent panels, the column labels indicate the value of $K_{\text {off }}$ in units of s ${ }^{-1}$. 

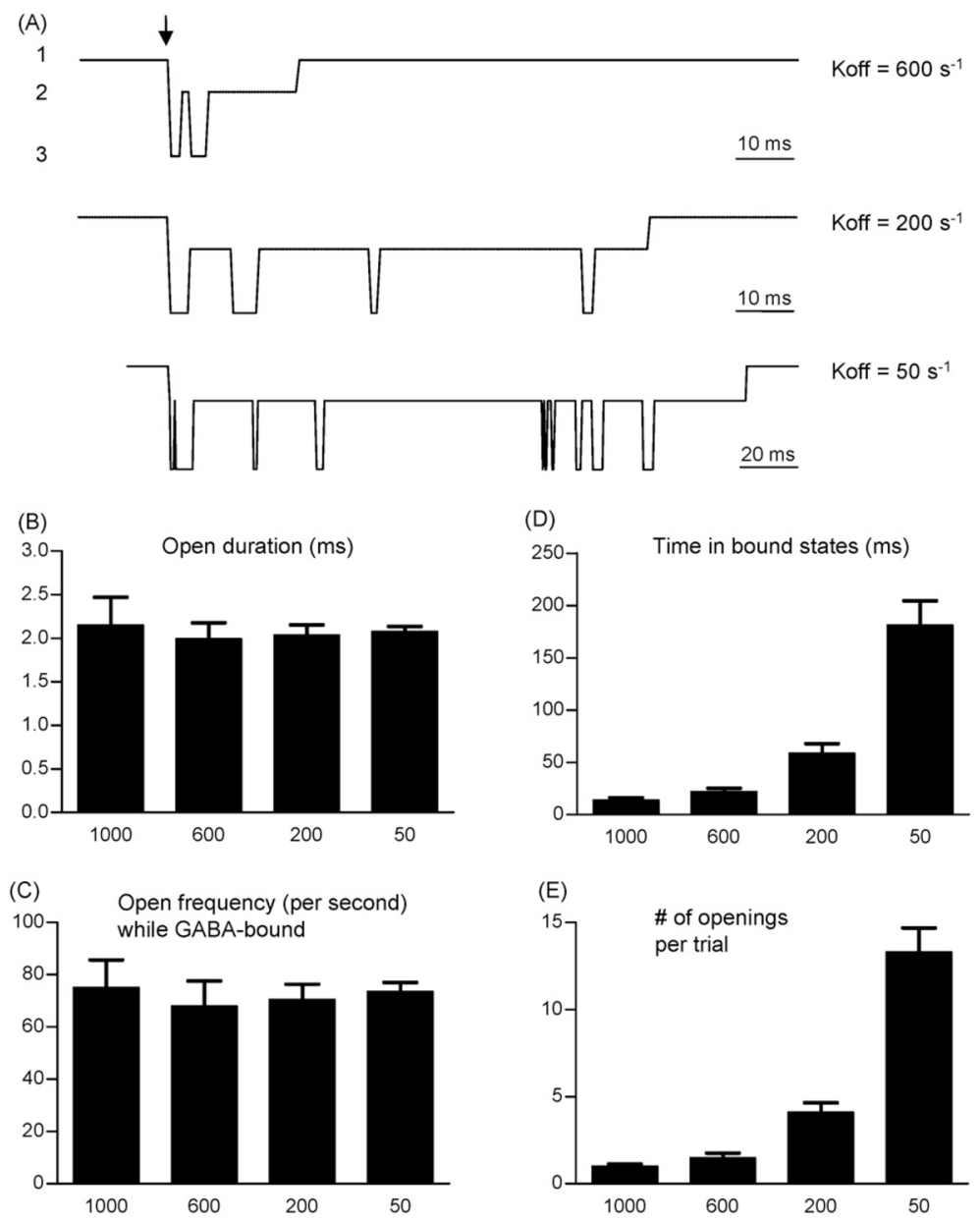

Figure 5.

Simulated single-channel activity under "synaptic" conditions. (A) Simulated single-channel activity in response to a $1 \mathrm{~ms}$ pulse of $1 \mathrm{mM}$ GABA when $K_{\text {off }}$ is $600 \mathrm{~s}^{-1}$ (top trace), $200 \mathrm{~s}^{-1}$ (middle trace), and $50 \mathrm{~s}^{-1}$ (bottom trace). The current levels designate receptor presence in the $C_{\mathrm{u}}$ (level 1), $C_{\mathrm{b}}$ or $D$ (level 2), and $O$ (level 3) states. (B) Mean open duration (ms) was not significantly altered by changes in $K_{\text {off }}$. In this and subsequent panels, the column labels indicate the value of $K_{\text {off }}$ in units of s ${ }^{-1}$. (C) Mean opening frequency, measured while channels were bound by GABA, was not significantly altered by changes in $K_{\text {off. }}$ (D) The time spent in the bound states increased as $K_{\text {off }}$ decreased, with significant differences in every pair-wise column comparison except between $K_{\text {off }}=1000$ and $K_{\text {off }}=600$. (E) The mean number of openings per trial pulse of GABA increased as $K_{\text {off }}$ decreased, with significant differences in comparison of every pair of columns except between $K_{\text {off }}=1000$ and $K_{\text {off }}=600$. 